\title{
If-Then Plans Benefit Delay of Gratification Performance in Children With and Without ADHD
}

\author{
Caterina Gawrilow • Peter M. Gollwitzer • \\ Gabriele Oettingen
}

\begin{abstract}
Children with impulse control deficits (i.e., children with ADHD) are known to have special problems with delaying gratifications. As making if-then plans (i.e., forming implementation intentions) has been found to benefit self-control even in individuals whose action control is chronically hampered (e.g., critical samples such as patients with frontal lobe damage, the elderly), we analyzed whether delay of gratification is facilitated in children with and without ADHD who have formed respective implementation intentions. In Study 1 , forty-five inpatient children with ADHD $\left(M_{\text {age }}=10.7\right.$ years $)$ increased delay of gratification performance after having formed respective implementation intentions. Study 2 replicated this finding in an outpatient sample of children with ADHD $(n=47$, $M_{\text {age }}=10.3$ years) and also in a comparison group of children without ADHD $\left(n=40, M_{\mathrm{age}}=11.3\right.$ years $)$.
\end{abstract}

\footnotetext{
C. Gawrilow ( $₫)$

German Institute for International Educational Research (DIPF), Center for Individual Development and Adaptive Education of Children at Risk (IDeA), University of Frankfurt, Mertonstr. 17, 60325 Frankfurt, Germany

e mail: gawrilow@dipf.de

P. M. Gollwitzer - G. Oettingen

New York University, New York, NY, USA

P. M. Gollwitzer

University of Konstanz, Konstanz, Germany

G. Oettingen

University of Hamburg, Hamburg, Germany
}

Results are discussed with respect to their implications for action control in children with ADHD as well as research on implementation intentions and delay of gratification.

Keywords If-then plans - Self-control - Delay aversion . Delay of gratification - ADHD

Self-control research deals with the broad question of when and how people fail to do what they want to do (e.g., eating healthy, preparing for an exam) but rather act impulsively (e.g., eating fatty instead of healthy food, watching TV instead of reading a textbook). This question has important implications for various areas of life (e.g., health, school, and work performance). However, there is surprisingly little theoretical consensus about the question of how selfcontrol is achieved best.

For instance, Baumeister and colleagues have proposed that exerting self-control requires the inhibition of impulsive behavior, such as when a child that has the goal to sit quietly and work on a task in her/his textbook has to inhibit the urge to walk around in the classroom and talk with her/ his classmates. Here, effective self-control can be viewed as willful and conscious acting that needs resources and effort (overview by Tangney et al. 2004). Or, Mischel and colleagues have suggested that actions can be instigated by either "hot" or "cool" systems. More precisely, Metcalfe and Mischel (1999) proposed a two-system framework containing a cool, cognitive "know" system that enhances self-control and a hot, impulsive "go" system that diminishes self-control. The assumption is that when the hot system is dominant, exposure to a hot stimulus will elicit the respective hot and impulsive response (Metcalfe and Mischel 1999). 


\section{Self-Control in Children with ADHD}

Children with Attention Deficit Hyperactivity Disorder $(\mathrm{ADHD})^{1}$ are known to be highly impulsive: They have a hard time waiting their turn (e.g., APA 1994), inhibiting responses (e.g., Nigg 2001; Oosterlaan and Sergeant 1996), and planning actions (e.g., Barkley 1997a; Frazier et al. 2004). This impulsivity leads to several detrimental effects: Children with ADHD show risky behavior (i.e., fast driving; Jerome et al. 2006), sensation seeking (Antrop et al. 2000), are responsive to immediate rewards (Douglas and Parry 1994), and are less able to delay gratification or resist temptation (Rodriguez et al. 1989).

Prominent theories on ADHD suggest that these symptoms arise from primary deficits in executive control processes. In general, executive functions are necessary for the fulfillment of various task demands. These mental control processes establish an appropriate set of goal-directed actions required to meet one's goals (e.g., Fischer et al. 2005; Seidman 2006). However, a more elaborated model of ADHD proposes a distinction between executive function and motivation control deficits in children with ADHD. According to the dual pathway model of behavior and cognition introduced by Sonuga-Barke (2002), ADHD may not only pertain to a dysregulation of thought and action pathway (DTAP) but also to a motivational style pathway (MSP). Both children with ADHD DTAP and ADHD MSP meet criteria for the ADHD combined subtype even though they are characterized by distinct symptoms, development, etiology, and cognitive profiles as described below.

The first pathway (ADHD DTAP) is manifested in a primary inhibitory dysfunction that is mediated by secondary cognitive and behavioral dysfunctions, which in turn lead to faulty task-engagement (e.g., deficits of setshifting, working memory) and to symptomatic behavior (e.g., inattentiveness, hyperactivity). ADHD DTAP seems furthermore etiologically caused by neurobiological risk factors. Indeed, several studies and meta-analyses observed severe cognitive impairments, executive function deficits, and especially inhibition deficits in children with ADHD (Oosterlaan and Sergeant 1996; Oosterlaan et al. 1998). The second pathway (ADHD MSP) is characterized by a dysregulation of reward mechanisms leading to a higher preference for immediate rewards in children with ADHD. As associative learning plays an important role in the development of ADHD MSP, it is linked to environmental instead of neurobiological risk factors. ADHD MSP relates

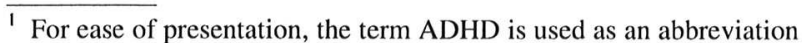
for Attention Deficit/Hyperactivity Disorder according to the ICD 10 category of Hyperkinetic Disorder and the DSM IV combined subtype.
}

empirically to research observing delay aversion and delay of gratification deficits in children with ADHD (SonugaBarke et al. 1992, 1996).

Indeed, experimental studies revealed that children with ADHD prefer an immediate but small reward over a delayed but bigger reward (Rapport et al. 1986; Scheres et al. 2008). Sonuga-Barke and colleagues used a delay aversion paradigm to investigate ADHD children's impulsivity (Choice Delay Task; Sonuga-Barke 2002; Sonuga-Barke et al. 2008): Children with ADHD waited less often than children without ADHD for a delayed reward when it was associated with a longer waiting period as compared to a shorter waiting period. Also, delay aversion measures appear to be stable over time in children with ADHD (Kuntsi et al. 2001). Furthermore, delay aversion correlates positively with teacher ratings of impulsivity, hyperactivity, and conduct problems. Delay aversion can therefore be seen as part of the ADHD symptomatology (Solanto et al. 2001). However, even though it is well established that children with ADHD are challenged in everyday and experimental situations that require selfcontrol in terms of delay aversion or delay of gratification, studies concerning the encouragement of self-control in those children are lacking (as pointed out by Reid et al. 2005; Strayhorn 2002).

\section{Enhancing Self-Control by Planning}

One proven strategy to facilitate self-control is planning and a particularly effective form of planning is forming implementation intentions. Implementation intentions (Gollwitzer 1993, 1999) take the format of "If situation X is encountered, then I will perform behavior Y!" and therefore link a critical situation (if-part) with a goaldirected response (then-part). It is important to recognize that implementation intentions differ from goal intentions: Goal intentions merely specify a desired outcome and have the format of "I intend to achieve Z!" While goal intentions specify preferred finite states (i.e., the performance of a desired behavior or the attainment of a desired outcome) that an individual feels committed to attain, implementation intentions predetermine how a specified critical situation will be responded to when it is encountered. Accordingly, if-then plans are subordinate to goal intentions and serve the purpose of enhancing effective goal striving.

Implementation intentions offer benefits beyond goal intentions: A meta-analysis by Gollwitzer and Sheeran (2006) involving more than 8,000 participants in 94 independent studies reported an effect size of $d=.65$. This medium-to-large effect size (Cohen 1992) represents the additional facilitation of goal achievement through if-then 
plans compared to goal intentions alone. As goal intentions by themselves already have a supporting effect on behavior enactment (Webb and Sheeran 2006), the size of this effect is remarkable. If-then plan effects are assumingly caused by different psychological processes: The mental links created by implementation intentions facilitate goal attainment on the basis of psychological processes that relate to both the anticipated situation (i.e., spelled out in the if-part of the plan) and the intended behavior (i.e., spelled out in the then-part of the plan). As forming an implementation intention implies the selection of a certain future situation, the mental representation of this situation becomes highly activated and hence more accessible (Gollwitzer 1999). This heightened accessibility of the situation specified in the if-part was observed in several studies (e.g., Aarts et al. 1999; Parks-Stamm et al. 2007; Webb and Sheeran 2007, 2008) meaning that people are more likely to identify and notice this situation when they subsequently encounter it (e.g., Webb and Sheeran 2004, 2007). Additionally, the specified situational cue automates the initiation of the intended behavior. Forming implementation intentions was found to facilitate immediate and efficient action initiation, not requiring a further conscious intent (e.g., Bayer et al. 2009; Brandstätter et al. 2001; Gollwitzer and Brandstätter 1997). Both mechanisms, the heightened accessibility of the specified situation and the automated initiation of the intended behavior, have been observed to mediate the if-then plan effects on behavior (Gollwitzer and Sheeran 2006).

\section{Planning and Self-Control in Children with ADHD}

Children with ADHD could benefit from the use of implementation intentions as a way to improve executive function problems and therefore possibly reduce difficulties with delay of gratification (Barkley 1997a; Nigg 2001; Sonuga-Barke 2002). This is because recent studies revealed that if-then plans support performance on tasks where challenges to executive functions are prevalent. Concretely, in task-shifting and conflict-management paradigms if-then plans improved performance beyond goal intentions in a college student sample (e.g., Cohen et al. 2008). Furthermore, the effect of if-then plans on a task assessing executive functions was evaluated in children with ADHD: Indeed, if-then plans helped children with ADHD when solving a Go/NoGo task. In these task children both classified stimuli (i.e., animals vs. transportation toys) that were presented on a computer screen by pressing a mapped computer key, as well as inhibited any classification in response to a NoGo signal (i.e., a sound). In two experiments (Gawrilow and Gollwitzer 2008), the authors randomly assigned children with ADHD to one of two conditions: Children in the goal intention condition formed a goal to inhibit a classification response for marked stimuli ("I will not press a key for pictures that have a sound"), while children in the implementation intention condition, in addition to forming a goal intention, formed an if-then plan ("And if I hear a sound, then I will not press any key"). In the first study, it was observed that children with ADHD who furnished a suppression goal with implementation intentions improved inhibition of an unwanted response on a NoGo signal trial to the same level observed in children without ADHD. The second study compared the performances of children with ADHD with and without psychostimulant medication and showed that a combination of if-then plans and psychostimulant medication resulted in the highest level of inhibition performance in children with ADHD (Gawrilow and Gollwitzer 2008).

Using the same task paradigm, Paul et al. (2007) measured electroencephalographic data of nonmedicated children with ADHD and control children in (a) a mere task instruction condition without a self-regulation strategy and (b) a condition that involved the making of if-then plans: The if-then plans did not only improved response inhibition but they also increased the NoGo-P300 in children with ADHD compared with the mere task instruction condition in this study. The NoGo-P300 represents response control and conflict monitoring, which are both reduced in untreated children with ADHD (Fallgatter et al. 2004). Apparently, the self-regulation strategy of forming if-then plans alters both behavioral and electrophysiological indices of performance in a Go/NoGo task among children with ADHD. Without this self-regulation strategy, children with ADHD made more inhibition errors following NoGo trials and had a significantly smaller NoGo Go amplitude difference than control children during the first half of the P300 component. No difference was observed between the control and ADHD groups when the children were given the self-regulation strategy. As the NoGo-P300 represents the endogenous evaluation of response control and conflict monitoring, these findings suggest that such processes become more pronounced when children with ADHD are given the self-regulation strategy of forming implementation intentions.

\section{Present Research}

In the present studies, we wanted to determine whether the action control facilitating effects of implementation intentions in children with ADHD (i.e., combined subtype) also hold for a typical measure of motivation control: delay of gratification (Chamberlain and Sahakian 2007). Note that our previous studies only showed that if-then plans prove effective concerning the alteration of inhibitory executive 
dysfunctions as one of the two pathways to ADHD (i.e., ADHD DTAP in the dual pathway model of ADHD by Sonuga-Barke 2002). The present studies are aimed at extending this finding to the second pathway of ADHD: the pathway of motivation control (i.e., ADHD MSP) as assessed in a delay aversion or delay of gratification paradigm. That implementation intentions have the potential to facilitate motivation control is indicated by prior research showing that even when people have an initial reluctance to engage in a certain behavior, they can facilitate its initiation by forming implementation intentions. Various studies observed that implementation intentions still manage to increase goal attainment rates when the respective goal is unpleasant to enact. For instance, the goal intentions to perform regular breast examinations (Orbell et al. 1997), cervical cancer screenings (Sheeran and Orbell 2000), resumption of functional activity after joint replacement surgery (Orbell and Sheeran 2000), and engaging in demanding physical exercise (Milne et al. 2002), were all more frequently acted upon when people had furnished these goals with implementation intentions.

Therefore, for the present two studies we hypothesized that forming implementation intentions should facilitate the unpleasant waiting that is demanded in a delay of gratification situation. In both studies, we compared the delay performance (i.e., waiting for a delayed reward) in a mere task instruction condition with both a goal intention condition and an implementation intention condition. Whereas only children with ADHD were participating in Study 1, a control group of children without ADHD was also participating in Study 2. For children with ADHD we expected delay facilitating effects of implementation intentions but not of goal intentions in both Studies 1 and 2. Furthermore, we expected that in children without ADHD not only implementation intentions but also goal intentions facilitate waiting in a delay of gratification task (Study 2). This is because waiting might be comparatively easier for children without ADHD so that goal intentions suffice to enhance waiting and implementation intentions are not needed.

\section{Study 1}

A self-developed computerized delay task was used in which participating children had to decide 40 times in a row between an immediate but small and a delayed but big reward. The incentive in this task was money, which was exchanged afterwards for points collected during the task. We decided to choose money as an incentive based on results of a pilot study in which we asked children with and without ADHD between 8 and 10 years of age whether they preferred a small gift with the value of $€ 5(\$ 7)$ or $€ 5$ (\$7) in cash. Ten of the 15 participating children in the pilot study decided that receiving cash would be their preference.

Participants in the main study were boys and girls with ADHD who were randomly assigned to three conditions (mere task instruction vs. goal intention vs. goal intention plus implementation intention). We hypothesized that the children would earn less money in the delay of gratification task if they received a mere task instruction without any goal. We expected furthermore that a mere goal intention would have no effect at all because delay of gratification is particularly difficult for children with ADHD and goal intentions have only minor effects on goal accomplishment in difficult tasks (Gollwitzer and Sheeran 2006). Hence, we hypothesized that goal intentions combined with if-then plans would support the children in tolerating longer waiting periods for bigger rewards.

\section{Method}

\section{Participants}

Forty-five children (14 girls) with ADHD $\left(M_{\text {age }}=\right.$ 10.73 years, $\mathrm{SD}=1.39$ ) participated in the study. All children were diagnosed with Hyperkinetic Disorder F90.0 (ICD-10; World Health Organization 1991) as their primary disorder and were sent to a hospital in Germany for an ADHD treatment, by the recommendation of the family's health insurance or their pediatrician. It is important to note that the hospital was not a psychiatric institution but an institution specialized in the treatment of ADHD (i.e., cognitive behavior therapy). Thirty children received medication with Methylphenidate (MPH). However, $48 \mathrm{~h}$ before and at the time of investigation participating children were not medicated with MPH or other substances. Furthermore, all children took part in the experiment during their first days on ward to prevent effects of cognitive behavioral therapy to be conducted on ward. With regard to ethnic background, all of the participating children were Caucasian. Exclusion criteria were comorbid disorders and medication with MPH at the time of investigation. The study is compliant with the 1964 World Medical Association Declaration of Helsinki and was approved by the responsible ethics committee.

\section{Procedure}

At their first 2 days on the ward, the children whose parents had given written informed consent to participate were scheduled for an appointment with the experimenter. At this meeting in a quiet room on the ward the computer task was explained. The experimenter described the rules of the 
task prior to the children answering several open-ended questions making sure that all children understood the task.

\section{Delay Task}

We decided to operationalize delay of gratification in line with paradigms used by Mischel $(1958,1974,1996)$ and Sonuga-Barke and colleagues (i.e., Sonuga-Barke 2002). Delay of gratification is traditionally measured in Walter Mischel's ingenious experimental set-up (Mischel 1958): Children are introduced to a decisional conflict before the experimenter leaves the room. Each child is shown a pair of rewards (e.g., treats such as snacks) that differ in value (e.g., one marshmallow vs. two). The children are told that to obtain the more precious reward they have will have to wait until the experimenter returns. However, the children are also told that they are free to end the waiting period by ringing a bell, but that by doing so they will get the less valuable reward and forgo the more precious one (summaries by Metcalfe and Mischel 1999; Mischel 1974; Mischel et al. 1989).

On the basis of experiments by Mischel and colleagues (e.g., Mischel et al. 1989) and studies by Sonuga-Barke (e.g., Sonuga-Barke et al. 1992), we developed a computerized delay of gratification task, where children had to decide 40 times between an immediate and small (red pictures showing means of transportation or animals with a value of one point) or a delayed and big gratification (blue pictures showing means of transportation or animals with a value of three points). The stimuli were presented on a 15 " computer screen. The earned points were counted in the upper left area of the computer screen. Additionally, a cash register sound appeared at the same time the children earned a point (i.e., one sound for one point and three sounds for three points). In the bottom area a growing bar told the children how many trials were completed and how many trials were left to perform. Additionally, the delay time was randomly varied between 30, 40, 50, and $60 \mathrm{~s}$ : Each of these four different delay intervals occurred ten times, respectively. A fixed, blocked randomization plan was used meaning that all children received the same randomized order of delay intervals divided into two parts (i.e., first and second 20 trials) that contained the same number of delay intervals, respectively. Red pictures and blue pictures appeared consecutively on the screen. Red pictures (one point) showed up first and after a delay of $30,40,50$, or $60 \mathrm{~s}$ blue pictures (three points) followed. Thus, the children had the opportunity to click onto the red picture and receive one point right away, or to wait $30,40,50$, or $60 \mathrm{~s}$, click onto the blue picture and receive three points. Every point the children earned during the computer task was equal to 5 cents the children would earn at the end of the computer task. Thus, children could earn between $€ 2$ and $€ 6$ (equivalent to approximately $\$ 2.60$ and $\$ 7.80$ ).

\section{Experimental Conditions}

The participants were randomly assigned to three conditions, which differed only in one sentence that children should remember while completing the task. One-third of the children received a mere task instruction ("Red pictures are one point, blue pictures are three points") and onethird of the children received a sentence containing a goal intention ("I will earn as many points as possible"). The remaining children received the goal intention and an additional implementation intention ("Whenever a red picture appears, then I will wait for the blue one"). Children had to repeat these sentences three times aloud. It is important to note that all participants received the same information about the task. The three conditions only varied in the phrasing of the sentences (above written in italics) that children were asked to keep in mind while completing the task.

\section{Interview}

At the end of the experiment, the experimenter conducted a short interview with each participant. We measured task commitment to ensure the equivalence of our sample concerning this variable in the three different conditions (i.e., mere task instruction, goal intention, and implementation intention) by asking participants to answer three items (e.g., "I intended to do well on the task," "It makes a difference for me to be good at this task," "I would have been very disappointed if I failed at this task") using a seven-point scale ranging from 1 (strongly agree) to 7 (strongly disagree).

\section{Design}

The study followed a 3-between (Condition: mere task instruction vs. goal intention vs. goal intention plus implementation intention) $\times$ 4-within (Delay Time: $30 \mathrm{~s}$ vs. $40 \mathrm{~s}$ vs. $50 \mathrm{~s}$ vs. $60 \mathrm{~s}$ ) design. The dependent variable was the amount of money the children earned altogether during the task.

Results

\section{Amount of Earned Money}

A 3-between (Condition: mere task instruction vs. goal intention vs. goal intention plus implementation intention) $\times$ 4-within (Delay Time: $30 \mathrm{~s}$ vs. $40 \mathrm{~s}$ vs. $50 \mathrm{~s}$ vs. $60 \mathrm{~s})$ repeated measurement ANOVA on the amount of 
Table 1 Means and standard deviations for amount of earned money (in $€$ ) in the delay task in children with ADHD by intention condition (Study 1) and in children with and without ADHD by intention condition (Study 2)

\begin{tabular}{|c|c|c|c|}
\hline \multirow{2}{*}{$\begin{array}{l}\text { Amount of earned } \\
\text { money }\end{array}$} & \multicolumn{3}{|l|}{ Condition } \\
\hline & $\begin{array}{l}\text { Mere task } \\
\text { instruction }\end{array}$ & $\begin{array}{l}\text { Goal } \\
\text { intention }\end{array}$ & $\begin{array}{l}\text { Implementatio } \\
\text { intention }\end{array}$ \\
\hline \multicolumn{4}{|l|}{ Study 1} \\
\hline Children with ADHD & $3.35(2.27)$ & $2.82(2.25)$ & $5.54(1.26)$ \\
\hline \multicolumn{4}{|l|}{ Study 2} \\
\hline Children with ADHD & $3.55(2.06)$ & $3.26(2.22)$ & $4.57(1.69)$ \\
\hline Children without ADHD & $3.54(2.21)$ & $4.01(2.01)$ & $4.76(1.81)$ \\
\hline
\end{tabular}

Standard deviations in parentheses

earned money revealed a significant main effect of Condition, $F(2,42)=7.69, P<.001$, no significant main effect of Delay Time, $F(2,42)=1.55$, NS, and no significant interaction effect of Condition and Delay Time, $F(2,42)=0.58$, NS. Specifically, participants waited less often for the delayed reward and therefore earned less money when they received a mere task instruction $(M=3.35 €, \mathrm{SD}=2.27)$ or a goal intention $(M=2.82 €$, $\mathrm{SD}=2.25)$ compared to a goal intention plus implementation intention $(M=5.54 €, \mathrm{SD}=1.26$; see Table 1) independent of Delay Time. Planned contrasts revealed a significant difference between the mere task instruction condition and the implementation intention condition, $t(42)=3.05, P<0.01$; a significant difference between the goal intention and the implementation intention condition, $t(42)=3.66, P<0.001$; but no significant difference between the mere task instruction condition and the goal intention condition, $t(42)=0.73$, NS. These results suggest that children with ADHD benefited from implementation intentions but not from mere task instructions or from goal intentions.

We also analyzed whether implementation intentions had beneficial effects over and above gender and age differences. Results indicated no significant effect of Gender, $F(1,41)=2.08$, NS, but a significant effect of Age so that children who were assigned to the implementation intention condition were younger than children in the other two conditions, $F(1,41)=14.23, P<.001$. However, when adding Age as a covariate, the main effect of Condition remained significant, $F(2,41)=6.98, P=.002$, indicating that the advantageous effect of if-then plans is independent of the participants' age.

\section{Influence of Task Commitment}

We summarized the three interview items to form one index assessing task commitment (Cronbach's $\alpha=.81$ ) and found no differences by Condition, $F(2,42)=0.38$,
NS. Thus, children who received a mere task instruction $(M=5.38, \quad \mathrm{SD}=1.43), \quad$ goal intention $(M=5.34$, $\mathrm{SD}=1.44)$, or implementation intention $(M=5.78$, $\mathrm{SD}=1.66)$ all revealed a task commitment on the same (high) level, indicating that the observed implementation intention effects were not due to changes in task commitment.

\section{Discussion}

Inpatient children with ADHD benefited from self-regulatory instructions in a delay of gratification task. However, the beneficial effect was visible only when the goal intention was combined with an implementation intention. A goal intention to do well produced no better effects than a mere instruction to perform the task. This was true despite the fact that the children of all experimental conditions showed a task commitment at the same high level.

Study 1 has several limitations. First, we did not assess intelligence and the socioeconomic status (SES) although both are important control variables. Thus, a screening of intelligence and measures of SES would be useful to check for the equivalence of the sample. Second, our sample consisted only of inpatient children; and third, we also did not include a control group of children without ADHD. These are shortcomings as the effectiveness of implementation intentions on delay of gratification performance should not only evince in this specific group of children with ADHD but also in children that suffer from ADHD but are not hospitalized and additionally in children that show no ADHD symptoms at all. To attend to these shortcomings, we ran a second study.

\section{Study 2}

In Study 2, we modified the delay of gratification task as the variation of the delay time did not have an effect in Study 1 . Hence, we altered the computerized delay of gratification task by omitting the delay time variation and setting the delay time at $30 \mathrm{~s}$ for each trial. Furthermore, we invited not only inpatient children with ADHD but we compared outpatient children with ADHD to children without any psychological disturbances. We expected a replication of the results found in Study 1 for children with ADHD: They should fail to improve delay of gratification performance with mere task instructions as well as with the use of goal intentions; but implementation intentions should be effective in children with ADHD. For children without ADHD we expected that goal intentions might already suffice to improve performance but that implementation intentions will definitely do so. This is because action control is quite intact in children without ADHD and 
thus for these children the task to be solved qualifies as easy. As a consequence, the delay task can be solved with the use of mere goal intentions and no additional implementation intentions are needed.

\section{Method}

\section{Participants}

Because gender had no effect on the results in Study 1 and the prevalence of $\mathrm{ADHD}$ is more frequent in males (Barkley 1990), we only included male children in our study. Eighty-seven boys participated $\left(M_{\text {age }}=10.34\right.$ years, $\mathrm{SD}=1.59$ ) whereby 47 boys were diagnosed with Hyperkinetic Disorder F90.0 (ICD-10; WHO 1991) as their primary disorder by the head pediatrician of a local pediatric clinic in Germany using semistructured interviews with the parents and the child, questionnaires for parents and teachers, and neuropsychological tests with the child. One-third of the children received medication with MPH. However, $48 \mathrm{~h}$ before and at the time of investigation participating children were not medicated with MPH or other substances. Furthermore, participating children had not received cognitive behavioral therapy during the last 6 months. Exclusion criteria were comorbid disorders and medication with MPH. The 40 control boys $\left(M_{\text {age }}=\right.$ 11.27 years, $\mathrm{SD}=1.44$ ) without $\mathrm{ADHD}$ were recruited from local schools in Germany. The study is compliant with the 1964 World Medical Association Declaration of Helsinki and was approved by the responsible ethics committee.

\section{Procedure}

Children and parents (after having given written informed consent) were scheduled for an appointment with the experimenter at the University. Upon arrival they were greeted at the laboratory (a quiet room) and introduced to the computer task. The experimenter described the rules of the task prior to the children answering several open-ended questions to make sure that all children understood the task.

\section{Delay Task}

In 40 trials, red and blue pictures appeared consecutively on the screen. The children had the option to immediately click onto the red picture and receive one point right away, or to wait $30 \mathrm{~s}$, click onto the blue picture and receive three points. Again, every point the children earned during the computer task was equivalent to 5 cents to be received at the end (i.e., children could earn between $€ 2$ and $€ 6)$.

\section{Experimental Conditions}

Children were randomly assigned to the same three conditions as in Study 1 (mere task instruction vs. goal intention vs. goal intention plus implementation intention) and we used the same questionnaire as in Study 1 to assess task commitment after finishing the task.

\section{Background Measures}

With regard to ethnic background, all of the children with and without ADHD were Caucasian. A SES index was obtained based on the parents' educational level and occupation. There was no significant difference between mothers of children with ADHD $(M=2.38, \mathrm{SD}=1.34)$ and without $\mathrm{ADHD}(M=2.88, \mathrm{SD}=1.00)$ concerning the SES index, $F(1,86)=1.61$, NS. However, there was a marginal difference between fathers of children with ADHD $(M=1.20, \quad \mathrm{SD}=0.69)$ and without ADHD $(M=1.60, \mathrm{SD}=0.72), F(1,86)=2.97, P=.06$ (see Table 2), indicating a higher educational level and better occupation in fathers of children without ADHD.

Parents of all participating children were asked to fill out the Child Behavior Check List (CBCL; Arbeitsgruppe Deutsche Child Behavior Checklist 1998) to measure different aspects concerning the children's behavior (e.g., social withdrawal, somatic disturbances, and anxiety/ depression in the internalizing scale; antisocial and aggressive behavior in the externalizing scale; social problems, schizoid/obsessive compulsive behavior and attention problems plus the aforementioned internalizing and externalizing scales in the total CBCL). The CBCL confirmed the diagnosis of the ADHD group in showing significant differences between children with ADHD $(M=67.36, \mathrm{SD}=6.76)$ and without ADHD $(M=53.02$, $\mathrm{SD}=8.77)$ concerning the total scale, $F(1,86)=60.97$, $P<.001$. Likewise the ratings concerning the internalizing

Table 2 Characteristics of the sample (Study 2)

\begin{tabular}{lccrrr}
\hline Variables & \multicolumn{2}{l}{ Children } & & \multirow{2}{*}{$F$} \\
\cline { 2 - 4 } & With ADHD & Without ADHD & & \\
\hline Age & $10.34(1.59)$ & $11.27(1.44)$ & 8.08 & .006 \\
Block design test & $9.83(2.64)$ & $10.78(2.82)$ & 2.69 & .10 \\
SES index mothers & $2.38(1.34)$ & $2.88(1.00)$ & 1.61 & .21 \\
SES index fathers & $1.20(0.69)$ & $1.60(0.72)$ & 2.97 & .06 \\
CBCL internalizing & $63.92(8.49)$ & $53.38(8.8)$ & 26.67 & .000 \\
CBCL externalizing & $63.50(11.77)$ & $52.14(8.83)$ & 21.01 & .000 \\
Total CBCL & $67.36(6.76)$ & $53.02(8.77)$ & 60.97 & .000
\end{tabular}

The block design test is from Tewes et al. (1999); the CBCL is from the Arbeitsgruppe Deutsche Child Behavior Checklist (1998). Stan dard deviations in parentheses 
scale differed significantly between children with ADHD $(M=63.92, \mathrm{SD}=8.49)$ and without ADHD $(M=53.38$, $\mathrm{SD}=8.8), F(1,86)=26.67, P<.001$, and so did the externalizing scale $(M=63.50, \mathrm{SD}=11.77$ vs. $M=$ 52.14, $\mathrm{SD}=8.83), F(1,86)=21.01, P<.001$ (Table 2). Hence, the ADHD group was in at risk or clinically significant ranges on the reported three scales of the CBCL, whereas the control group was in a normative range for all three scales.

Furthermore, all participating children had to solve one subtest from the intelligence test HAWIK-III (German version of the Wechsler Intelligence Scale for Children, WISC-III; Tewes et al. 1999) as a screening for intelligence. The block design test was chosen because of its high predictive and diagnostic value (Renner 2002; Sattler 1992). Children with ADHD $(M=9.83, \mathrm{SD}=2.64)$ and without ADHD $(M=10.78, \mathrm{SD}=2.82)$ did not differ significantly concerning their performance in the block design test, $F(1,86)=2.69$, NS (Table 2). Both groups, children with and without ADHD were average on the block design subtest relative to a norm (Tewes et al. 1999).

\section{Design}

The study followed a 3-between (Condition: mere task instruction vs. goal intention vs. goal intention plus implementation intention) $\times 2$-between factorial (Group: with ADHD vs. without ADHD) design. The dependent variable was the amount of money children earned altogether during the task

\section{Results}

Because the age of the children differed between groups (with ADHD: $M=10.34$, SD $=1.59$ vs. without ADHD: $M=11.27, \mathrm{SD}=1.44), F(1,86)=8.08, P<.05$, Age was used as a covariate in the analyses reported below.

\section{Amount of Earned Money}

A 3 (Condition: mere task instruction vs. goal intention vs. goal intention plus implementation intention) $\times 2$ (Group: with ADHD vs. without ADHD) ANOVA on amount of earned money revealed a significant main effect of Condition, $F(2,86)=3.72, P<.05$, no significant effect of Group, $F(2,86)=.02$, NS, and no significant interaction effect, $F(2,86)=.50$, NS. Apparently, all children (with and without $\mathrm{ADHD}$ ) earned the smallest amount of money in the mere task instruction condition $(M=3.57 €$, $\mathrm{SD}=2.11)$ closely followed by the goal intention condition $(M=3.61 €, \mathrm{SD}=2.11)$; only in the implementation intention condition a considerably larger amount was earned $(M=4.56 €, \mathrm{SD}=1.75)$.
Indeed, the planned contrast between the mere task instruction and the implementation intention conditions revealed that children with and without ADHD in the implementation intention condition acquired significantly more money than children in the mere task instruction condition, $t(55)=2.07, P<.05$. The planned contrast between the goal and the implementation intention conditions was also significant, $t(55)=2.19, P<.05$, again indicating that children in the implementation intention condition acquired comparatively more money. However, there was no significant difference between the mere task instruction and the goal intention conditions, $t(55)=.11$, NS.

Looking separately at children with and without ADHD, we observed that children with ADHD in the implementation intention condition $(M=4.57 €, \mathrm{SD}=1.69)$ earned more money than those in the mere task instruction condition $(M=3.55 €, \mathrm{SD}=2.06), t(29)=1.49, P<.05$, and those in the goal intention condition $(M=3.26 €$, $\mathrm{SD}=2.22), t(29)=1.83, P<.05$. The same was true for children without ADHD (implementation intention: $M=4.76 €, \mathrm{SD}=1.8$; mere task instruction: $M=3.54 €$, $\mathrm{SD}=2.2$; goal intention: $M=4.01 €, \mathrm{SD}=2.0$; see Table 1, Study 2). However, inspection of the means suggests for children without ADHD that they already benefited from goal intentions and not only from implementation intentions. Therefore, we tested whether children without ADHD in the goal intention condition earned more money than those in the mere task instruction condition whereas in the implementation intention condition earned the most. In fact, the test of a linear trend, with the amount of earned money being higher in the implementation intention condition compared to the goal intention condition compared to the mere task instruction condition was significant in the group of children without ADHD, $z=1.23, n=40, P=.04$; whereas the same test for a linear trend was not significant in the group of children with ADHD, $z=0.53, n=47, P=.33$.

\section{Influence of Background Variables}

We also analyzed the influence of the assessed background variables: SES index, behavioral ratings, and intelligence screening. The main effect (reported above) did not change significantly when entering the SES index of the father as a covariate variable and correlational analyses suggested that performance in the delay of gratification task was not significantly related to the SES index, $r(84)=.04$, NS. Furthermore, the observed pattern of results remained the same after covarying out CBCL ratings (total $T$ value) and correlational analyses indicated that performance in the delay of gratification task was not significantly related to CBCL ratings, $r(84)=-.08$, NS. However, within cell correlations 
further suggest that the CBCL externalizing scale rating was significantly related to delay of gratification in children with and without ADHD in the mere task instruction condition, $r(28)=-.64, P<.01$, and goal intention condition, $r(27)=-.54, P<.05 ;$ the correlation was not significant in the implementation intention condition, $r(26)=.09$, NS. Thus, only in the if-then plan condition problematic externalizing behavior (as indicated in parental ratings) was not associated with delay of gratification performance.

Lastly, the main effect did not change significantly when entering the scores derived from the intelligence screening as a covariate variable, even though there was a significant positive correlation between the amount of money earned and the intelligence screening measure, $r(84)=.35$, $P<.001$. However, within cell correlations suggest that IQ scores significantly correlated with delay of gratification in the mere task instruction, $r(27)=.45, P<.01$, and the goal intention condition, $r(29)=.38, P<.05$; the correlation was not significant in the implementation intention condition, $r(26)=.32, P=.11$. Thus, only in the if-then plan condition performance in an intelligence screening was not associated with delay of gratification performance.

These additional analyses suggest that the observed benefit from implementation intentions in children with and without ADHD is independent of SES, behavioral ratings, and intelligence.

\section{Influence of Task Commitment}

We summarized the three items to form one index assessing task commitment (Cronbach's $\alpha=.75$ ) and found no main effect of Condition, $F(2,77)=0.49$, NS, and no interaction effect of Condition and Group, $F(2$, $77)=0.42$, NS. Thus, children with and without ADHD who received a mere task instruction $(M=5.4$, $\mathrm{SD}=0.65)$, goal intention $(M=5.27, \mathrm{SD}=0.87)$, or implementation intention $(M=5.19, \quad \mathrm{SD}=0.83)$ all reported a similarly high task commitment. However, the main effect of Group was marginally significant, $F(2$, $77)=3.28, P=.07$, indicating that children with ADHD reported a higher task commitment $(M=5.43, \mathrm{SD}=0.72)$ compared to children without ADHD $(M=5.11$, $\mathrm{SD}=0.83$ ). This pattern of data suggests that the observed changes in delay of gratification caused by implementation intentions are not due to an increase in task commitment. This conclusion is also supported by the fact that level of commitment did not significantly correlate with delay of gratification performance, $r(80)=.04$, NS.

\section{Discussion}

Both children with and without ADHD benefited from forming implementation intentions in a task requiring delay of gratification. Specifically, children who formed an ifthen plan could delay gratification better during the task and therefore earned more money at the end of the task than children who received a mere task instruction or a goal intention to do well on the task. Children without ADHD already benefited from goal intentions (and even more from implementation intentions), but children with ADHD needed implementation intentions to improve their delay of gratification performance.

\section{General Discussion and Implications}

In line with the general prediction, our studies indicate that goal intentions endowed with implementation intentions are superior to overcome self-control problems in a delay of gratification task in participants with and without ADHD. Children with ADHD markedly increased their performance from the goal intention to the implementation intention condition. A meta-analysis of the goal intention versus implementation intention effect in children with ADHD of Studies 1 and 2 taken together indicates that the effect size is large $(d=1.088, N=60, k=2)$, homogenous (Chi-square $(1)=.092, \mathrm{NS})$, and significant $(95 \%$ $\mathrm{CI}=-15.89$ to $18.06, P=.001)$.

\section{Implications for ADHD Research}

The two studies suggest that implementation intentions are not only beneficial regarding executive functions as shown by Gawrilow and Gollwitzer (2008) but also facilitate motivation control in children with ADHD. In the present Studies 1 and 2, goal intentions seemed not to be superior compared to mere task instructions in children with ADHD: Explicitly setting the goal to obtain more points and therefore a bigger reward was not helpful for delaying gratifications in children with ADHD. In comparison, children without ADHD showed linear improvements over the three conditions (i.e., mere task instruction vs. goal intention vs. implementation intention) and therefore already benefited from a goal intention. Delay of gratification seems to be a difficult to attain goal for children with ADHD (Sonuga-Barke 2002) and thus needs the support of if-then plans.

Furthermore, that children with ADHD did not benefit from instructions used in the goal intention condition might indicate that they have a harder time following nonspecific goals formulated in vague directions (at least more so than children without ADHD). This could be an important reason for the effectiveness of current ADHD behavioral treatment programs targeting the cognitive-behavioral training of parents (Anastopoulos et al. 2006; Barkley 1997b): For instance, in these training programs parents 
learn strategies to attend to child behavior as well as strategies to manage child behavior (i.e., responding immediately and consistently, providing precise instructions). However, this conclusion is tentative and further research should investigate this hypothesis in more detail. Teaching different behavior management strategies to parents of children with ADHD and comparing the effects of parents' assigning goal versus implementation intentions on the child's everyday behavior should reveal interesting insight.

\section{Implications for Treating Disorders of Self-Regulation}

Years ago, Meichenbaum and colleagues successfully instructed impulsive children to use private speech (e.g., "Good, I'm doing fine so far. Remember go slow!"; Meichenbaum and Goodman 1971). Impulsive children who participated in this self-instruction training committed fewer errors. These results contributed an important detail to self-regulation research that is in line with the present findings: children with self-regulation difficulties can benefit from self-instructions. Since Meichenbaum's research was published, only a few studies examined the effectiveness of self-instructions in children with ADHD (overview by Harris et al. 2005). Our studies extend existing findings suggesting that implementation intention self-instructions should be superior to goal intention selfinstructions. Thus, teaching children with ADHD to tackle problems of impulsivity by forming implementation intentions might be an important supplement to existing therapeutic programs. Additionally, if-then plans might also have an impact on day-to-day self-regulation problems these children are facing (i.e., studying for tests, household tasks and rules). Thus, further research might want to explore whether forming if-then plans can be taught to children with ADHD as a meta-cognitive strategy that they then can apply on their own in everyday life.

\section{Implications for Implementation Intention Research}

Past research on implementation intentions focused on various self-regulatory problems that prevent people from reaching their goals (i.e., failing to get started, getting derailed, not calling a halt, or overextending oneself) and results showed that if-then plans help people to overcome these problems (Gollwitzer and Sheeran 2006). For instance, if-then plans are found to be effective regarding common self-regulatory deficits in everyday live (e.g., eating a healthy diet, using dental floss). Most relevant to the present research, if-then plans were also found to support performance on tasks where challenges to motivation control are prevalent: when people have to engage in behaviors that are quite unpleasant (e.g., taking certain medical tests) and thus are reluctant to do so.
Research further showed that if-then plans are helpful for people facing chronic self-regulation problems: In the past, implementation intention research with clinical samples has been conducted to test process hypotheses on how if-then plans achieve their effects. For instance, the hypothesis that if-then plans lead to efficient action control that does not suffer from cognitive load was tested by assessing whether also heroine addicts during withdrawal and patients suffering from schizophrenia benefit from forming if-then plans (Brandstätter et al. 2001). Indeed, in populations that are burdened by self-regulatory shortcomings, if-then plans turned out to be equally (or even more) effective as in control samples (e.g., college students).

The present studies are of theoretical significance in the field of implementation intention research for the following reasons: First, the studies showed again that populations suffering from self-regulation deficits (i.e., children with ADHD) still benefit from if-then plans (Gawrilow and Gollwitzer 2008; Paul et al. 2007). Second, the present studies replicated and extended existing research on the effectiveness of if-then plans in children with ADHD: Whereas prior studies focused on performances implicating executive functions (i.e., Go/NoGo task; Gawrilow and Gollwitzer 2008; Paul et al. 2007), the present studies analyzed motivation control (i.e., performance in a delay of gratification task): Again, implementation intentions displayed their beneficial effects on action control. This is of particular importance as possessing delay of gratification skills is linked to a variety of positive, long-term outcomes (e.g., academic performance; Eigsti et al. 2006; Shoda et al. 1990).

\section{Implications for Delay of Gratification Research}

The present studies may further be seen as supporting the hot/cool system model (Metcalfe and Mischel 1999), which involves a cool, cognitive know system that enhances selfcontrol and a hot impulsive go system that diminishes selfcontrol. When the hot system is dominant (e.g., when the cool system is chronically dysfunctional as is the case in children with ADHD), exposure to the hot stimulus will elicit the respective hot response (Metcalfe and Mischel 1999). Implementation intentions however, enable children with ADHD to change their impulsive way of going for an immediate reward by strengthening the waiting response. Thus, by forming implementation intentions children with ADHD can strengthen the cool system and therefore longer waiting periods for delayed rewards are observed.

Teaching children the strategy of forming implementation intentions as a simple self-regulatory tool might therefore be an important supplement to existing therapeutic programs. Still, further research is needed to 
investigate the effectiveness of implementation intentions as a separate component of ADHD interventions such as cognitive behavioral training programs that focus on behavioral parent training and behavioral interventions in the classroom (e.g., Pelham et al. 1998).

\section{Limitations of the Studies}

In the mere task instruction condition of Study 2, we could not find any significant difference between children with and without ADHD concerning their delay of gratification performance as measured by the amount of earned money. One explanation might be that the differences in delay of gratification performance of children with and without ADHD are not reflected in the main dependent outcome variable (i.e., earned money). This interpretation is in line with recent research by Eigsti et al. (2006) showing that it is not the frequency of waiting for a delayed reward but the behavior during the delay of gratification situation (e.g., focusing the attention away from the reward) at preschool age that is the most powerful predictor of cognitive skills (i.e., performance in a $\mathrm{Go} / \mathrm{NoGo}$ task) 15 years later. Therefore, one limitation of the present studies might be that we measured only the amount of earned money in the delay of gratification task. Further research should also include observations of the children's behavior while waiting.

A second explanation of the missing difference between children with and without ADHD might be the use of a delay aversion paradigm. Sonuga-Barke and colleagues characterize within the framework of their Delay Aversion Hypothesis impulsive behavior "not as the consequence of a relative inability to inhibit a response, but rather the result of a rational choice to avoid delay, which the individual finds aversive" (Solanto et al. 2001, p. 217). Thus, SonugaBarke and colleagues assume that children with ADHD are not impaired in delay of gratifications per se but only try to avoid delay time. The authors further state that reward parameters (i.e., very attractive, valuable rewards) exert little control over the behavior of a child with ADHD; nevertheless, they recognize that all children will be somehow sensitive to changes in reward size to some extent. It might be the case therefore that the external reward (i.e., money) used in our studies was too attractive for children with ADHD and therefore the task was too easy to produce differences between children with and without ADHD.

Concerning the difficulty of delaying gratification, Mischel and colleagues observed that the presence of the actual rewards during the delay period makes delay of gratification more difficult, whereas the absence of the actual rewards during the delay period or attention to their symbolic representations in the form of images (i.e., framed pictures of the rewards) facilitates delay (Yates and Mischel 1979). Thus, to produce differences between children with and without ADHD, future studies might want to vary the difficulty of the used delay of gratification task. This is possible by varying both the quality of the rewards and the way in which they are presented (i.e., rewards with different values, material and immaterial rewards, presence and absence of rewards).

The difficulty explanation of the missing differences regarding delay of gratification performance of children with and without ADHD in Study 2 is partly supported by the data collected in our two studies: Comparing the mean amount of earned money in the mere task instruction conditions of inpatient children with ADHD (Study 1), outpatient children with ADHD (Study 2), and children without ADHD (Study 2, see Table 1) suggests that inpatient children have the strongest difficulties in delaying gratification. As inpatient children with ADHD are commonly characterized by more symptom-related problems compared to outpatient children and to children without ADHD, this result supports the difficulty hypothesis. Furthermore, Scheres et al. (2008) pointed out that missing differences between children with and without ADHD in a delay aversion paradigm could be due to a predominance of participating ADHD children with the inattentive subtype because delay aversion is mostly associated with hyperactivity-impulsivity symptoms and not with inattentiveness (Scheres et al. 2006). A significant negative correlation of the CBCL externalizing scale rating and earned money in the mere task instruction and goal intention conditions in Study 2 supports this assumption. The more externalizing problem behavior parents observed in their children, the less delay of gratification their children showed in our delay of gratification task in the mere task instruction condition, $r(28)=-.64$, $P<.01$.

But could implementation intentions still benefit the delay of gratification performance of children with ADHD if the difficulty of the delay task is increased? Research has shown that participants with implementation intentions outperform participants with goal intentions more drastically when the task at hand is difficult rather than easy to solve (summary by Gollwitzer and Sheeran 2006). For instance, Gollwitzer and Brandstätter (1997) observed that university students benefited more from implementation intentions with respect to completing a difficult as compared to an easy personal goal and Lengfelder and Gollwitzer (2001) found that patients with a frontal lobe injury benefited more from forming implementation intentions than a comparison group of university students when performing a medium difficult Go/NoGo task. This suggests that children with ADHD should benefit most from implementation intentions when they need them, that is, 
when the delay of gratification task to be performed becomes more difficult.

Another limitation of Study 2 is that the participating groups of children (with ADHD vs. without ADHD) were not matched concerning age, intelligence, and SES; they differed significantly in age and the means showed a trend towards a significant difference in intelligence. Although intelligence seemed to have no influence on implementation intention effects in children with ADHD, it would be compelling for future research to assemble matched groups of children with and without ADHD. In the same vein, taking executive function measures and motivation control measures would be useful (a) to employ executive functions and motivation control as a further matching variable and (b) to explore whether children with motivation control problems benefit more from implementation intentions in delay of gratification tasks than children with executive function deficits.

An additional limitation concerns the generalizability of the present findings. In both studies children with ADHD were not allowed to have any comorbid disorders. Therefore, the beneficial effect of if-then plans on delay of gratification performance might not apply to children with ADHD who also show comorbid disorders. As children with ADHD frequently suffer from comorbid psychiatric disorders (Fischer et al. 2005), future research should make an effort to recruit both children with and children without comorbid disorders.

A final limitation concerns the task-specific information given in different instructions of both studies (i.e., mere task instruction, goal intention, goal intention plus implementation intention). Specifically, the sentences used in the two intention conditions (i.e., "I will earn as many points as possible" as a goal intention and "Whenever a red picture appears, then I will wait for the blue one" as an implementation intention) seem to differ regarding the amount of strategy-related information that is given with respect to performing the task at hand. Past research on implementation intentions has dealt with this problem by enriching the goal intention with the more detailed information provided in the implementation intention (e.g., Bayer and Gollwitzer 2007) or by striping the implementation intention of the more detailed strategy information (e.g., Henderson et al. 2008). No matter which approach is taken, implementation intentions led to superior goal attainment as compared to goal intentions.

\section{Conclusion}

The main finding of the two studies reported is that boys and girls with and without ADHD benefit from forming implementation intentions for delaying gratification. Thus, children can increase delay of gratification performance by if-then plans that specify when and where they intend to do what in order to facilitate unpleasant waiting. This selfregulation strategy to enhance motivation control is simple, as it only requires forming and committing to a straightforward plan.

\section{References}

Aarts, H., Dijksterhuis, A. P., \& Midden, C. (1999). To plan or not to plan? Goal achievement of interrupting the performance of mundane behaviors. European Journal of Social Psychology, 29, 971979.

American Psychiatric Association. (1994). Diagnostic and statistical manual of mental disorders (4th ed.). Washington, DC: Amer ican Psychiatric Association.

Anastopoulos, A. D., Rhoads, L. H., \& Farley, S. E. (2006) Counseling and training parents. In R. A. Barkley (Ed.), ADHD: $A$ handbook for diagnosis and treatment (3rd ed., pp. 453 479). New York: Guilford Press.

Antrop, I., Roeyers, H., Van Oost, P., \& Buysse, A. (2000). Stimulation seeking and hyperactivity in children with ADHD. Journal of Child Psychology and Psychiatry and Allied Disci plines, 41, 225231.

Arbeitsgruppe Deutsche Child Behavior Checklist. (1998). Eltern fragebogen uber das Verhalten von Kindern und Jugendlichen. Deutsche Bearbeitung der Child Behavior Checklist (CBCL/4 18) [German consensus version of the Child Behavior Checklist (CBCL/4 18)]. Köln: Arbeitsgruppe Kinder, Jugend und Familiendiagnostik (KJFD).

Barkley, R. A. (1990). Attention deficit/hyperactivity disorder: A handbook for diagnosis and treatment. New York: Guilford.

Barkley, R. A. (1997a). Behavioral inhibition, sustained attention, and executive functions: Constructing a unifying theory of ADHD. Psychological Bulletin, 121, 6594.

Barkley, R. A. (1997b). Defiant children: A clinician's manual for assessment and parent training. New York: Guilford.

Bayer, U. C., Achtziger, A., Gollwitzer, P. M., \& Moskowitz, G. (2009). Responding to subliminal cues: Do if then plans cause action preparation and initiation without conscious intent? Social Cognition, 27, 183201.

Bayer, U. C., \& Gollwitzer, P. M. (2007). Boosting scholastic test scores by willpower: The role of implementation intentions. Self and Identity, 6, 119.

Brandstätter, V., Lengfelder, A., \& Gollwitzer, P. M. (2001). Implementation intentions and efficient action initiation. Journal of Personality and Social Psychology, 81, 946960.

Chamberlain, S. R., \& Sahakian, B. J. (2007). The neuropsychiatry of impulsivity. Current Opinion in Psychiatry, 20, 255261.

Cohen, J. (1992). A power primer. Psychological Bulletin, 112, 155 159.

Cohen, A. L., Bayer, U. C., Jaudas, A., \& Gollwitzer, P. M. (2008). Self regulatory strategy and executive control: Implementation intentions modulate task switching and Simon task performance. Psychological Research, 72, 1226.

Douglas, V. I., \& Parry, P. A. (1994). Effects of reward and nonreward on frustration and attention in attention deficit disorder. Journal of Abnormal Child Psychology, 22, 281302.

Eigsti, I., Zayas, V., Mischel, W., Shoda, Y., Ayduk, O., Dadlani, M. B., et al. (2006). Predictive cognitive control from preschool to late adolescence and young adulthood. Psychological Science, 17,478484 . 
Fallgatter, A. J., Ehlis, A. C., Seifert, J., Strik, W. K., Scheuerpflug, P., Zillessen, K. E., et al. (2004). Altered response control and anterior cingulate function in ADHD boys. Clinical Neurophys iology, 115, 973981.

Fischer, M., Barkley, R. A., Smallish, L., \& Fletcher, K. (2005). Executive functioning in hyperactive children as young adults: Attention, inhibition, response perseveration, and the impact of comorbidity. Developmental Neuropsychology, 27, 107133.

Frazier, T. W., Demaree, H. A., \& Youngstrom, E. A. (2004). Meta analysis of intellectual and neuropsychological test performance in attention deficit hyperactivity disorder. Neuropsychology, 18 543555.

Gawrilow, C., \& Gollwitzer, P. M. (2008). Implementation intentions facilitate response inhibition in children with ADHD. Cognitive Therapy and Research, 32, 261280.

Gollwitzer, P. M. (1993). Goal achievement: The role of intentions. European Review of Social Psychology, 4, 141185.

Gollwitzer, P. M. (1999). Implementation intentions. Strong effects of simple plans. American Psychologist, 54, 493503.

Gollwitzer, P. M., \& Brandstätter, V. (1997). Implementation intentions and effective goal pursuit. Journal of Personality and Social Psychology, 73, 186199.

Gollwitzer, P. M., \& Sheeran, P. (2006). Implementation intentions and goal achievement: A meta analysis of effects and processes. Advances of Experimental Social Psychology, 38, 69119.

Harris, K. R., Danoff Friedlander, B., Saddler, B., Frizzelle, R., \& Graham, S. (2005). Self monitoring of attention versus self monitoring of academic performance: Differential effects among students with ADHD in the regular classroom. Journal of Special Education, 39, 145156.

Henderson, M. D., Gollwitzer, P. M., \& Oettingen, G. (2008). Implementation intentions and disengagement from a failing course of action. Journal of Behavioral Decision Making, 20, 81102.

Jerome, L., Segal, A., \& Habinski, L. (2006). What we know about ADHD and driving risk: A literature review, meta analysis and critique. Journal of the Canadian Academy of Child and Adolescent Psychiatry, 15, 105125.

Kuntsi, J., Stevenson, J., Oosterlaan, J., \& Sonuga Barke, E. J. S. (2001). Test retest reliability of a new delay aversion task and executive measures. British Journal of Developmental Psychol ogy, 19, 339348.

Lengfelder, A., \& Gollwitzer, P. M. (2001). Reflective and reflexive action control in patients with frontal brain lesions. Neuropsy chology, 15, 80100.

Meichenbaum, D. H., \& Goodman, J. (1971). Training impulsive children to talk to themselves: A means of developing self control. Journal of Abnormal Psychology, 77, 115126.

Metcalfe, J., \& Mischel, W. (1999). A hot/cool system analysis of delay of gratification: Dynamics of willpower. Psychological Review, 106, 319

Milne, S., Orbell, S., \& Sheeran, P. (2002). Combining motivational and volitional interventions to promote exercise participation: Protection motivation theory and implementation intentions. British Journal of Health Psychology, 7, 163184.

Mischel, W. (1958). Preference for delayed reinforcement: An experimental study of a cultural observation. Journal of Abnor mal and Social Psychology, 56, 5761.

Mischel, W. (1974). Processes in delay of gratification. In L. Berkowitz (Ed.), Advances in social psychology (pp. 249 292). New York: Academic Press.

Mischel, W. (1996). From good intentions to willpower. In P. M. Gollwitzer \& J. A. Bargh (Eds.), The psychology of action: Linking cognition and motivation to behavior (pp. 197 218). New York: Guilford Press.
Mischel, W., Shoda, Y., \& Rodriguez, M. L. (1989). Delay of gratification in children. Science, 244, 933938.

Nigg, J. T. (2001). Is ADHD a disinhibitory disorder? Psychological Bulletin, 127, 571598.

Oosterlaan, J., Logan, G. D., \& Sergeant, J. A. (1998). Response inhibition in $\mathrm{ADHD}, \mathrm{CD}$, comorbid $\mathrm{ADHD}+\mathrm{CD}$, anxious and normal children: A metaanalysis of studies with the stop task. Journal of Child Psychology and Psychiatry, 39, 411425.

Oosterlaan, J., \& Sergeant, J. A. (1996). Inhibition in ADHD, aggressive, and anxious children: A biologically based model of child psychopathology. Journal of Abnormal Child Psychology, 24,1936 .

Orbell, S., Hodgins, S., \& Sheeran, P. (1997). Implementation intentions and the theory of planned behavior. Personality and Social Psychology Bulletin, 23, 945954.

Orbell, S., \& Sheeran, P. (2000). Motivational and volitional processes in action initiation: A field study of the role of implementation intentions. Journal of Applied Social Psychol ogy, 30, 780797

Parks Stamm, E. J., Gollwitzer, P. M., \& Oettingen, G. (2007). Action control by implementation intentions: Effective cue detection and efficient response initiation. Social Cognition, 25, 248266

Paul, I., Gawrilow, C., Zech, F., Gollwitzer, P. M., Rockstroh, B., Odenthal, G., et al. (2007). If then planning modulates the P300 in children with attention deficit hyperactivity disorder. Neuro Report, 18, 653657.

Pelham, W. E.,. Wheeler, T., \& Chronis, A. (1998). Empirically supported psychosocial treatments for attention deficit hyperac tivity disorder. Journal of Clinical Child Psychology, 27, 190 205

Rapport, M. D., Tucker, S. B., DuPaul, G. J., Merlo, M., \& Stoner, G. (1986). Hyperactivity and frustration: The influence of control over size of rewards in delay of gratification. Journal of Abnormal Child Psychology, 14, 191204.

Reid, R., Trout, A. L., \& Schartz, M. (2005). Self regulation interventions for children with ADHD. Exceptional Children, 71,361377

Renner, G. (2002). Kritische Werte und Konfidenzintervalle zur Interpretation von HAWIK III IQ Skalen, Index Werten und Subtests [Differences required for significance and confidence intervals to aid the interpretation of HAWIK III IQ scores, index scores and subtests]. Zeitschrift fur Differentielle und Diagnostische Psychologie, 23, 353358.

Rodriguez, M. L., Mischel, W., \& Shoda, Y. (1989). Cognitive person variables in the delay of gratification in older children at risk. Journal of Personality and Social Psychology, 57, 358367.

Sattler, J. M. (1992). Assessment of children's intelligence. San Diego: Academic Press.

Scheres, A., Dijkstra, M., Ainslie, E., Balkan, J., Reynolds, B., Sonuga Barke, E., et al. (2006). Temporal and probabilistic discounting of rewards in children and adolescents: Effects of age and ADHD symptoms. Neuropsychologia, 44, 20922102.

Scheres, A., Lee, A., \& Sumiya, M. (2008). Temporal reward discounting and ADHD: Task and symptom specific effects. Journal of Neural Transmission, 115, 221226.

Seidman, L. J. (2006). Neuropsychological functioning in people with ADHD across the lifespan. Clinical Psychology Review, 26, 466485 .

Sheeran, P., \& Orbell, S. (2000). Using implementation intentions to increase attendance for cervical cancer screening. Health Psychology, 18, 283289.

Shoda, Y., Mischel, W., \& Peake, P. (1990). Predicting adolescent cognitive and self regulatory competencies from preschool delay of gratification: Identifying diagnostic conditions. Developmen tal Psychology, 26, 978986. 
Solanto, M. V., Abikoff, H., Sonuga Barke, E. J. S., Schachar, R., Logan, G. D., Wigal, T., et al. (2001). The ecological validity of delay aversion and response inhibition as measures of impulsivity in ADHD: A supplement to the NIMH multimodal treatment study of ADHD. Journal of Abnormal Child Psychology, 29, 215228.

Sonuga Barke, E. J. S. (2002). Psychological heterogenity in ADHD A dual pathway model of behavior and cognition. Behavioral Brain Research, 130, 2936

Sonuga Barke, E. J. S., Sergeant, J. A., Nigg, J., \& Willcutt, E. (2008). Executive dysfunction and delay aversion in ADHD: Nosological and diagnostic implications. Child and Adolescent Psychiatric Clinics of North America, 17, 367384.

Sonuga Barke, E. J. S., Taylor, E., Sembi, S., \& Smith, J. (1992). Hyperactivity and delay aversion $\mathrm{I}$. The effect of delay on choice. Journal of Child Psychology and Psychiatry, 33, 387398.

Sonuga Barke, E. J. S., Williams, E., Hall, M., \& Saxton, T. (1996). Hyperactivity and delay aversion III. The effect on cognitive style of imposing delay after errors. Journal of Child Psychology and Psychiatry, 37, 189194.

Strayhorn, J. M. (2002). Self control: Toward systematic training programs. Journal of the American Academy of Child and Adolescent Psychiatry, 41, 1727.

Tangney, J. P., Baumeister, R. F., \& Boone, A. (2004). High self control predicts good adjustment, less pathology, better grades, and interpersonal success. Journal of Personality, 72, 271324.
Tewes, U., Rossmann, P., \& Schallberger, U. (1999). Wechsler intelligence test for children, HAWIK III. Bern: Huber.

Webb, T. L., \& Sheeran, P. (2004). Identifying good opportunities to act: Implementation intentions and cue discrimination. European Journal of Social Psychology, 34, 407419.

Webb, T. L., \& Sheeran, P. (2006). Does changing behavioral intentions engender behavior change? A meta analysis of the experimental evidence. Psychological Bulletin, 132, 249268.

Webb, T. L., \& Sheeran, P. (2007). How do implementation intentions promote goal attainment? A test of component processes. Journal of Experimental Social Psychology, 43, 295302.

Webb, T. L., \& Sheeran, P. (2008). Mechanisms of implementation intention effects: The role of goal intentions, self efficacy, and accessibility of plan components. British Journal of Social Psychology, 47, 373395.

WHO. (1991). Internationale Klassifikation psychischer Storungen: ICD 10, Kapitel $V$. [Tenth revision of the international classification of diseases, Chapter $V(F)$ : Mental and behavioral disorders]. Bern: Huber.

Yates, B. T., \& Mischel, W. (1979). Young children's preferred attentional strategies for delaying gratification. Journal of Personality and Social Psychology, 37, 286300. 